\title{
¿Perdidos en la maleza? Una guía exhaustiva de malas hierbas no autóctonas en Florida ${ }^{1}$
}

\author{
Deah Lieurance, Lyn A. Gettys, y Germán Sandoya-Miranda²
}

Florida es una zona crítica para las especies invasoras no autóctonas tanto a escala nacional como mundial. Dichas especies son una amenaza para el medio ambiente, la economía y la salud humana. Como los investigadores y administradores de tierras en Florida llevamos décadas haciendo frente a las especies invasoras. Actualmente, el público tiene a su disposición numerosos recursos sobre especies invasoras. Sin embargo, la cantidad de información disponible podría resultar confusa. Existen cinco listas principales diferentes de especies no autóctonas que se detallan a continuación: 1. La Lista Federal de Malas Hierbas Nocivas, 2. La Lista de Florida de Malas Hierbas Nocivas, 3. La Lista de Plantas Acuáticas Prohibidas en Florida, 4. La Lista del Consejo de Plantas Exóticas de Florida (FLEPPC, por sus siglas en inglés) y 5 . La Evaluación de Plantas No Autóctonas del Instituto de Alimentos y Ciencias Agrícolas de la Universidad de la Florida (UF/IFAS por sus siglas en inglés). Este artículo tiene como objetivo informar al público en general, administradores de tierras, investigadores, autoridades locales y estatales, y a todo aquel que busque orientación para acceder a listados de plantas no autóctonas en el estado de Florida, tanto normativos como no normativos. En este documento se explica el origen de las listas, el significado de la inclusión en una lista en concreto y la forma de acceder a cada una de ellas.
Las especies invasoras no autóctonas suponen una amenaza significativa para el medio ambiente debido a la degradación de ecosistemas, la reducción de la diversidad de las especies y la economía dado a la pérdida de ingresos en la agricultura y el sector de recreación, y el elevado costo que conlleva controlar la invasión de estas poblaciones. Florida es especialmente susceptible a invasiones biológicas debido a su clima tropical/subtropical, a su forma peninsular y al número de especies introducidas manera intencionada (mediante cultivos hortícolas y agrícolas) y no-intencionada (a través del turismo y el comercio).

Los investigadores y administradores de tierras llevan muchos años haciendo frente a las especies de plantas invasoras. De hecho, el Centro para Especies de Plantas Acuáticas e Invasoras de UF/IFAS se fundó en el año 1979. Debido al hecho que el estado de la Florida lleva lidiando muchos años con problemas asociados a las especies invasoras, el estado cuenta con una gran cantidad de recursos específicos sobre especies invasoras (por ejemplo, identificación, gestión, alternativas a las especies invasoras). Particularmente, el estado de Florida cuenta con cinco listas primarias que catalogan las especies no autóctonas, las plantas invasoras y muchas otras listas menores (por ejemplo, Listas de Reacción Rápida para Detección Precoz de Especies Invasoras en Florida/Florida Invasive Species

1. Este documento, SS-AGR-436-Span, (The English version of this document is Lost in the Weeds?: A Comprehensive Guide to Florida's Many Non-Native Plant Lists (SS-AGR-436)) es uno de una serie de publicaciones del Departamento de Agronomía, Servicio de Extensión Cooperativa de la Florida, Instituto de Alimentos y Ciencias Agrícolas, Universidad de la Florida (UF/IFAS Extension). Fecha de primera publicación: noviembre 2020. Visite nuestro sitio web EDIS en <https://edis.ifas.ufl.edu>.

2. Deah Lieurance, científica asistente de Extensión y coordinadora de UF/IFAS Assessment, el Departamento de Agronomía; Lyn A. Gettys, profesora asociada de agronomía, UF/IFAS Fort Lauderdale Research and Education Center; y Germán Sandoya-Miranda, profesor asistente, Horticultural Sciences Department, UF/IFAS Everglades REC; UF/IFAS Extension, Gainesville, FL 32611.

The Institute of Food and Agricultural Sciences (IFAS) is an Equal Opportunity Institution authorized to provide research, educational information and other services

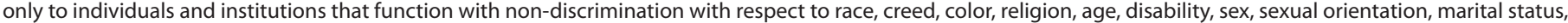

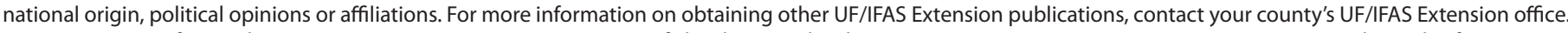
U.S. Department of Agriculture, UF/IFAS Extension Service, University of Florida, IFAS, Florida A \& M University Cooperative Extension Program, and Boards of County Commissioners Cooperating. Nick T. Place, dean for UF/IFAS Extension. 
Partnership Early Detection Rapid Response lists, Guía de Plantas de Paisajismo Aptas para Florida de la Universidad de Florida/UF's Florida-Friendly Landscaping ${ }^{\text {tm }}$ Plant Guide, y Descripciones Informativas de Especies Invasoras en Áreas Naturales de Florida/Florida Natural Areas Inventory Invasive Species Information Descriptions). Este documento hace hincapié en cinco listas primarias que son aplicables a Florida y tiene como objetivo evitar confusión sobre el carácter de estas listas, el significado de la inclusión en una lista en particular y la manera de acceder a cada una de ellas.

\section{La Ley Federal de Malas Hierbas Nocivas (FNWA,} por sus siglas en inglés), aprobada en 1975, creó un programa federal para el control de la propagación Malas Hierbas Nocivas en Estados Unidos. La FNWA otorga al Secretario de Agricultura la competencia para catalogar plantas como "Malas Hierbas Nocivas," creando así la Lista Federal de Malas Hierbas Nocivas (Federal Noxious Weed List). Una vez que una planta se incluye en dicha lista, queda prohibido todo movimiento interestatal o internacional de cualquier parte viva de las especies en lista (salvo que se cuente con un permiso), limitando por tanto el transporte de las mismas entre estados. La FNWA se modificó en 1990 para incluir disposiciones exigiendo a las agencias federales de administración de tierras (por ejemplo, la Agencia de Administración del Territorio, el Servicio de Parques Nacionales, el Servicio de Pesca, Fauna y Flora estadounidense) que tomasen medidas para reducir la propagación de especies invasoras. En el año 2000, la mayoría de la FNWA se incorporó en la Ley de Protección de Plantas, que es la legislación más reciente que regula las especies invasoras a escala nacional. A pesar de todas estas modificaciones $\mathrm{y}$ actualizaciones, el transporte interestatal de las especies de esta lista sigue estando prohibido sin el permiso del Departamento de Agricultura de los Estados Unidos, Servicio de Inspección de Salud Animal y Vegetal, Protección y Cuarentena Fitosanitarias (United States Department of Agriculture, Animal and Plant Health Inspection; USDA APHIS PPQ, por sus siglas en inglés). Las sanciones para quienes incumplen la ley incluyen el decomiso del material vegetal y la imposición de multas por delito menor (Oficina de Publicaciones del Gobierno de los Estados Unidos, 2019).

La Lista Federal de Malas Hierbas Nocivas está a cargo del USDA APHIS PPQ y la última actualización se produjo en 2010 (en 2017 se efectuaron cambios menores). La lista incluye diversas especies que se encuentran en Florida, incluidas el carrizo (Imperata cylindrica), la hydrilla (Hydrilla verticillata), el helecho trepador del viejo mundo (Lygodium microphyllum), tropical soda apple (Solanum viarum), melaleuca (Melaleuca quinquenervia) y el helecho Acuático (Salvinia molesta) (Figura 1). Para más información, puede consular el Tratado de Plantas Protegidas en línea: https:// www.govinfo.gov/content/pkg/PLAW-106publ224/html/ PLAW-106publ224.htm.

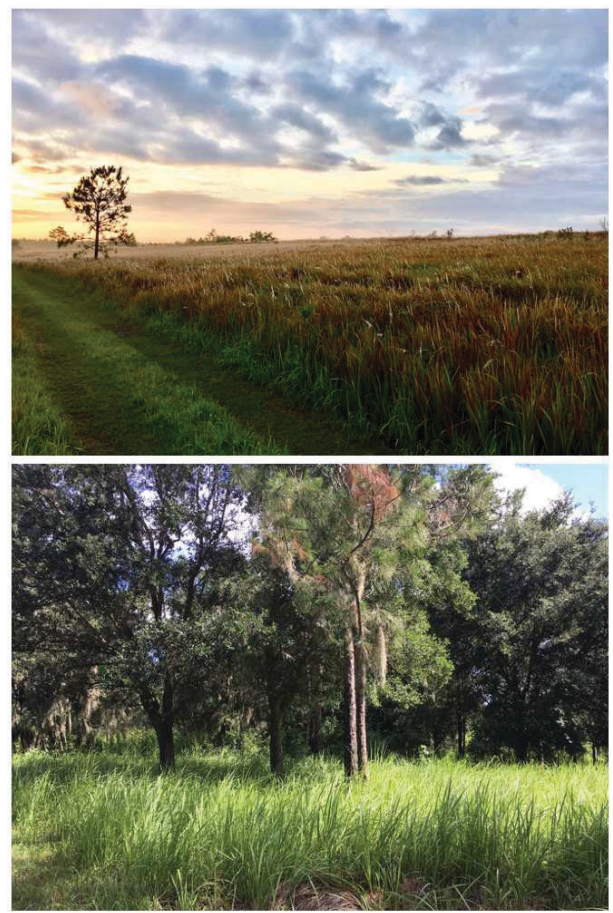

Figura 1. Dos ejemplos de invasión de carrizo (Imperata cylindrica) en el centro de la Florida, la primera en un campo al aire libre y la segunda en un área natural forestal. El ñame blanco (conocido con otros nombres anteriormente descritos) se encuentra en la Lista Federal de Malas Hierbas Nocivas. Está prohibido todo transporte interestatal o internacional de esta especie.

Créditos: Drew Hiatt y Taylor Clark, UF/IFAS

\section{La Lista de Malas Hierbas Nocivas de Florida es el} equivalente específico para el estado de la Lista Federal de Malas Hierbas Nocivas. Se creó en 1993 y se ha modificado en diversas ocasiones para añadir nuevas especies identificadas como invasoras. El Departamento de Agricultura y Servicios al Consumidor de Florida (FDACS, por sus siglas en inglés) supervisa esta lista (incluyendo la adición de nuevas especies y las exenciones para cultivos agronómicos y hortícolas de especies de la lista); la División de la Industria Vegetal del FDACS (DPI, por sus siglas en inglés) aplica la normativa (inspecciones y multas). De manera similar a las especies de la lista federal, "es ilegal introducir, reproducir, poseer, transportar o liberar cualquier hierba nociva" sin el permiso respectivo (FDACS 2019a). El FDACS define una hierba nociva como: 
"Cualquier forma viviente de un parásito u otra planta que pueda representar una amenaza seria para la agricultura en Florida; que pueda tener un impacto negativo sobre especies vegetales en vías de extinción, amenazadas o explotadas comercialmente; o si la planta es una planta naturalizada que altera comunidades de plantas autóctonas que se producen de forma natural" (FDACS 2019b).

Esta lista incluye tres géneros de malas hierbas parásitas (Aeginetia spp., Alectra spp., y la no nativa Cuscuta spp.) y más de 70 especies que incluyen ñame blanco, que también se conoce como yampí, mapuey, ñame baboso, papañame y papa de aire (Dioscorea bulbifera) (Figura 2), pimentero brasileño (Schinus terebinthifolius), ardisia (Ardisia crenata) y peladera (Leucaena leucocephala). Tradicionalmente, la Lista de Malas Hierbas Nocivas de Florida se ha limitado a especies de tierras altas, pero ahora se han incluido en esta lista especies acuáticas invasoras tales como las gencianas acuáticas con dos especies (Nymphoides cristata and $N$. peltata). Se puede acceder a la lista completa en el siguiente enlace.

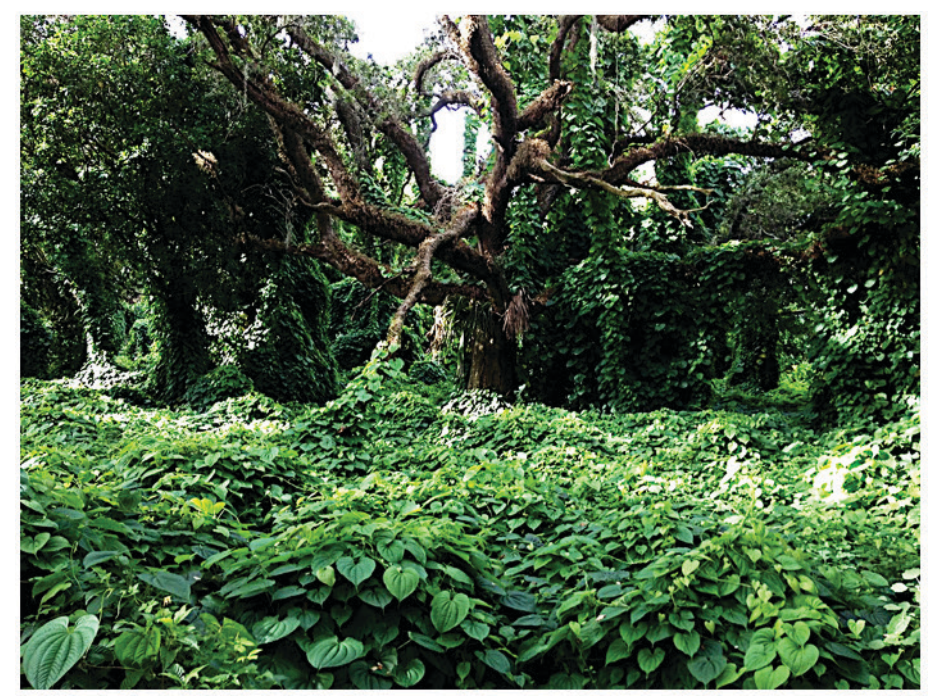

Figura 2. Invasión de ñame blanco (también conocido como, yampí, ñame blanco, ñame baboso, papañame y papa de aire) (Dioscorea bulbifera) en el sur de la Florida (Condado de Broward). El ñame blanco está en la Lista de Malas Hierbas Nocivas de Florida. EI FDACS DPI monitorea y ejecuta esta lista.

Créditos: Deah Lieurance, UF/IFAS

\section{La Lista de Plantas Acuáticas Prohibidas en Florida} se elaboró en 1986 y se modificó en 1993. Inicialmente, esta lista la administraba el Departamento de Protección Ambiental de Florida (DEP, por sus siglas en inglés), pero actualmente se encuentra bajo la administración de FDACS. De acuerdo con la norma 5B-64 de FDACS, "la Agencia de Inspección de Plantas y Apiarios del Departamento de Agricultura y de Servicios al Consumidor deberá administrar el programa de manejo de plantas acuáticas del estado, ya que podría ser necesario erradicar, controlar o la impedir la introducción y propagación de plantas acuáticas nocivas o prohibidas a través de la importación, transporte, el cultivo fuera de vivero, colecta, venta o posesión de plantas acuáticas" (FDACS 2019c). Existen dos categorías de la Lista de Plantas Acuáticas Prohibidas en Florida: Plantas Acuáticas prohibidas de Categoría I, que se adhieren a las mismas leyes aplicables a las plantas de la Lista de Malas Hierbas Nocivas de Florida, pero las plantas de la Categoría II Plantas se pueden cultivar en viveros, siempre y cuando dispongan de un permiso concedido por el estado y solo vendan las plantas a clientes fuera del estado de Florida. Al igual que sucede con las plantas de las Listas Malas Hierbas Nocivas Federal y de Florida, es ilegal cultivar, vender, transportar o comerciar con plantas de Categoría I (FDACS 2019c). Las sanciones por incumplir esta ley incluyen el decomiso y la destrucción sin compensación de cualquier planta regulada y la revegetación donde la excesiva recolección haya tenido como consecuencia ha provocado la disminución en su hábitat. Los infractores podrían ser declarados culpables de un delito menor de segundo grado y multados.

Una serie de plantas de Categoría I (que incluye el pino Australiano [Casuarina spp.], la melaleuca y la hydrilla) también se encuentran en la Lista Federal de Malas Hierbas Nocivas. Sin embargo, algunas especies de Categoría I son problemáticas solamente en Florida, entre ellas la lagunilla (Alternanthera philoxeroides) y los jacintos de agua (Eichhornia crassipes) (Figura 3). Se puede acceder a la lista de plantas acuáticas de Florida se puede acceder en el siguiente enlace.

\section{El Consejo de Plagas Vegetales Exóticas de Florida} (FLEPPC, por sus siglas en inglés), una organización sin fines de lucro que se constituye en 1984, ofrece una lista de las plantas más invasoras en Florida. El objetivo del FLEPPC es "respaldar la gestión de plantas exóticas invasoras en áreas naturales de Florida proporcionando un foro para el intercambio de información científica, educativa y técnica." Una de las maneras de fomentar este objetivo es a través de la Lista de plantas del FLEPPC. El Comité de Listas de Plantas (formado por biólogos, botanistas, ecologistas y administradores de tierras que regularmente consideran la adición de especies nuevas y cualquier cambio en el estatus de especies invasoras ya incluidas) desarrolla y mantiene esta lista. La lista FLEPPC no es reguladora, pero se ha incorporado a muchos de los procedimientos de gestión de plantas invasoras a nivel estatal y regional. Los listados se basan en información 
científica y observación de campo. Primero el Comité de Listas de Plantas y después el Consejo de Administración deben alcanzar un consenso antes de que una especie se añada oficialmente a la lista. Esta lista se revisa cada dos años.

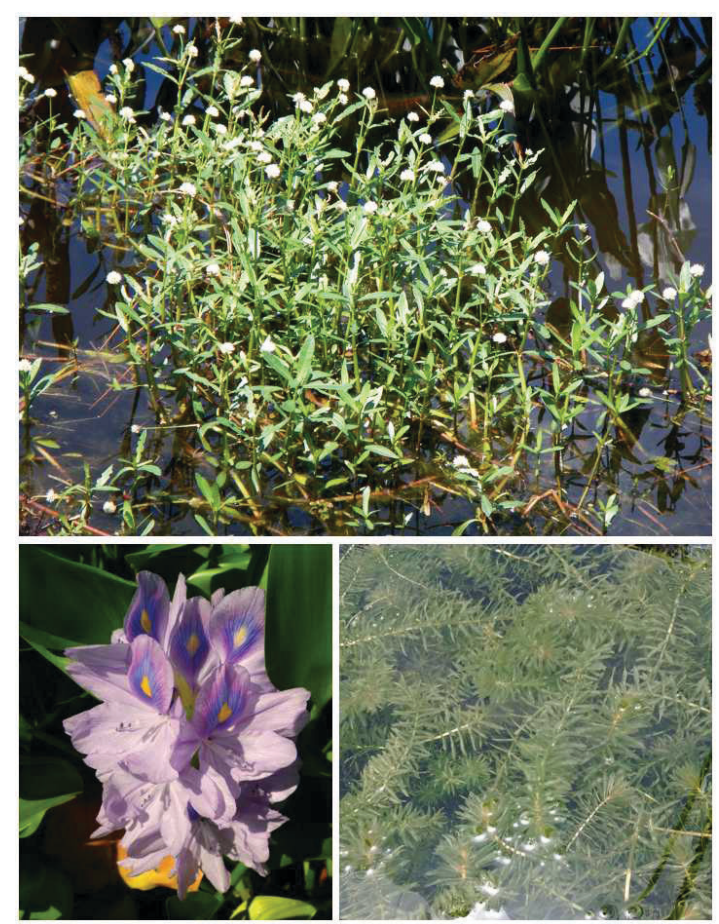

Figura 3. Lagunilla (Alternanthera philoxeroides), jacinto de agua (Eichhornia crassipes), y hydrilla (Hydrilla verticillata). Las tres especies están incluidas en la Lista de Plantas Acuáticas Prohibidas de Florida pero también están incluidas en la Lista Federal de Malas Hierbas Nocivas. EI FDACS DPI controla y hace cumplir la Lista de Plantas Acuáticas Prohibidas en la Florida.

Créditos: Lyn A. Gettys, UF/IFAS

Las especies en la Lista de Plantas del FLEPPC se agrupan en dos categorías, basadas en el nivel de impacto ecológico. Las plantas invasoras de la Categoría I son aquellas que alteran "comunidades de plantas autóctonas al reemplazar especies autóctonas, modificando las estructuras de las comunidades o las funciones ecológicas, o hibridándose con especies nativas" (FLEPPC 2019). Esta conclusión, se basa en evidencias documentadas de daños ecológicos y no en la gravedad económica o el alcance geográfico de la invasión. La Categoría II de especies invasoras son plantas que han "aumentado en abundancia o frecuencia, pero no han alterado aún las comunidades de plantas en Florida al mismo nivel que la Categoría I" (FLEPPC 2019). Estas especies podrían ascender a Categoría I si se vuelven más invasivas o causan más daños ecológicos. Muchas de estas especies están en la lista del FLEPPC y también en las listas federales y estatales (indicadas en la columna "Lista Gub."). La lista incluye una columna denominada "Zona” para documentar en qué lugar una especie podría causar un mayor impacto (norte, centro o sur de Florida).
5. La Evaluación de Plantas No Autóctonas en Áreas Naturales de Florida de UF/IFAS (en lo sucesivo, Evaluación UF/IFAS) utiliza herramientas de análisis basadas en la bibliografía sobre el tema para evaluar la situación de invasión o el riesgo de especies no autóctonas presentes en el estado, nuevas especies cuya introducción se ha propuesto, y nuevas selecciones agronómicas y hortícolas, híbridos y cultivares. La “evaluación de situación" (SA, por sus siglas en inglés) se utiliza para evaluar especies actualmente presentes en el estado e incorpora información (impactos ecológicos y dificultad de gestión) de expertos (administradores de tierras, científicos, etc.) para completar esta evaluación. La "herramienta predictiva" (PT, por sus siglas en inglés) es un modelo de valoración de riesgo que se utiliza para predecir el potencial o el riesgo de una especie de convertirse en invasora en el estado. Los cultivares se evalúan con el Protocolo de Taxonomía Intraespecífica (ITP, por sus siglas en inglés), siendo el mejorador genético que desarrolla el cultivar quien suministra los datos. Los resultados de estos análisis se utilizan en el proceso de liberación de un cultivar en UF/IFAS. Al contrario de las listas anteriores, la Evaluación UF/IFAS ofrece conclusiones para especies no autóctonas, tanto invasoras como no invasoras (Figura 4). La Evaluación UF/IFAS ofrece las siguientes recomendaciones:

- Si la especie no se considera un problema, podría recomendarse (SA, ITP)

- Riesgo de invasión reducido, podría recomendarse (PT)

- Precaución, podría recomendarse, pero se exige una plan para prevenir escapes (SA, ITP)

- Evaluación mas ampliar, puede ser recomendada, pero se exige plan para prevenir escapes (PT)

- Invasiva y no recomienda (SA, ITP)

- Alto riesgo y no se recomienda (PT)

De vez en cuando se dan circunstancias en las que el Grupo de Trabajo para Plantas invasoras de UF/IFAS podría desarrollar "Usos específicos y limitados" para especies invasoras o de alto riesgo. En estos casos específicos, se podrían recomendar estas especies siguiendo las directrices (ver conclusiones para palmas cocoteras en el sur de Florida). Además, en las bases de datos existen especies que se han evaluado con más de una de las herramientas recomendadas. En estos casos, se incluyen varias conclusiones. Sin embargo, las instrucciones indican que se debe seguir la conclusión más conservadora. 


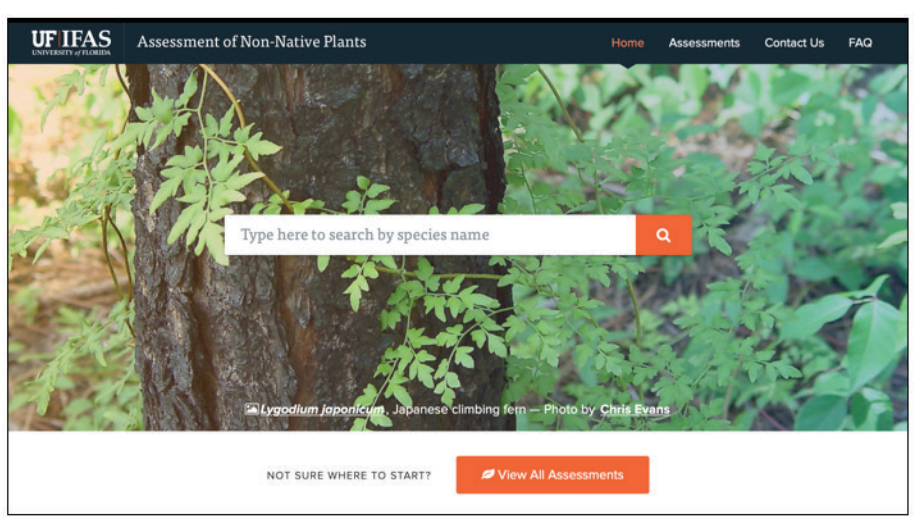

Figura 4. La página web de la Evaluación UF/IFAS donde se pueden encontrar los resultados de la evaluación de 900 especies no autóctonas. La lista incluye especies "invasivas" o "de alto riesgo de invasión," "precaución (requiere gestión para prevenir escapes)" o "evaluación adicional" y "recomendación aceptable o de bajo riesgo de invasión." La lista de Evaluación UF/IFAS ofrece información detallada sobre la situación de una especie (https://assessment.ifas.ufl.edu). Créditos: UF/IFAS

Aunque las conclusiones de la Evaluación UF/IFAS no tienen carácter reglamentario para el público en general, los investigadores y otro personal de UF/IFAS no están autorizados a recomendar el uso de especies "invasoras" o "de alto riesgo." El público también puede utilizar las conclusiones no invasoras (por ejemplo, "No se considera especie problemática por el momento" y "especie de bajo riesgo") en las bases de datos para crear una lista permisible de plantas que debería integrarse en el paisaje y podría ser cultivada. Esto no garantiza que una especie en particular no se vuelva invasora en el futuro, por lo que la Evaluación UF/IFAS regularmente revalúa especies para documentar cualquier cambio de comportamiento. En la actualidad constan 900 especies en las bases de datos de la Evaluación UF/IFAS. Si se detecta la ausencia de especies no autóctonas en las bases de datos, se puede solicitar una evaluación en la página web. Para más información sobre el funcionamiento de la Evaluación UF/IFAS y qué tipo de datos se utilizan para llegar a conclusiones, se puede acceder en el siguiente enlace web https://edis.ifas.ufl.edu/ag376.

\section{Conclusiones}

El estado de la Florida es una zona crítica para especies no autóctonas tanto a nivel nacional como internacional. Existen muchos recursos a disposición del público sobre especies invasoras, ya que los investigadores y administradores de tierras llevan décadas haciendo frente a las especies invasoras. El acceso a estos recursos resulta confuso y abrumador para el público en general. El estado de la Florida cuenta con cinco listas primarias que clasifican plantas invasoras no autóctonas; no se puede generalizar diciendo que una planta en particular está "en la lista." Tres de las cinco listas que se utilizan para clasificar plantas no autóctonas en Florida tienen carácter normativo (la Lista Federal de Malas Hierbas Nocivas, la Lista de Malas Hierbas Nocivas de Florida y La Lista de Plantas Acuáticas Prohibidas en Florida) y dos (Lista de Plantas FLEPPC y Evaluación UF/IFAS) realizan recomendaciones en base a conocimiento disponibles sobre distribución, impactos ecológicos y dificultad de gestión. Consulte la Tabla 1 para más información sobre la situación de las normativas, quienes (usuarios) deberían utilizarlas y enlaces a las listas de plantas. Las especies descritas en las Categorías I y II que se recogen en la lista de plantas FLEPPC y las especies con las calificaciones "invasiva" o "de alto riesgo" y "precaución" o "de riesgo moderado/evaluar" que se incluyen en la Evaluación UF/IFAS deberían evitarse debido a su naturaleza invasora o potencialmente invasora. Es fundamental escoger la clasificación adecuada cuando se trata con especies no autóctonas. El uso correcto de estas listas puede reducir la invasión de especies no autóctonas en Florida y en todo el sureste de los Estados Unidos. Para más información sobre especies no nativas, contacte con su agente de Extensión local de UF/IFAS.

\section{Glosario}

- Especies no autóctonas-Hace referencia a las especies (o subespecies, cultivares, selecciones, etc.) introducidas (intencionalmente o por accidente) fuera de su pasada y distribución actual (sinónimos: exótico, foráneo).

- Especies naturalizadas-Una planta no autóctona que no necesita de la ayuda humana para reproducirse y mantenerse por sí misma a lo largo del tiempo fuera de su hábitat (sinónimo: casual).

- Especie invasiva-Una especie no autóctona (o foránea) para el ecosistema bajo consideración cuya introducción causa o probablemente cause daños económicos y ambientales o perjudique la salud humana. Las especies invasoras pueden ser plantas, animales y cualquier otro organismo (por ejemplo, microbios).

- Malas hierbas-Una mala hierba es una planta (autóctona o no) que carece de valor en el lugar donde crece. Cualquier planta que represente una amenaza para la agricultura y/o ecosistemas naturales en Estados Unidos.

- Malas hierbas nocivas-Contexto legal (Ley Federal de Protección de Plantas): "Cualquier planta o producto vegetal que puede causar daños directos e indirectos a cultivos (incluidos viveros o productos vegetales), ganado, aves de corral u otros productos agrícolas, riego, navegación, los recursos naturales de Estados Unidos, la 
salud pública o el medio ambiente" (Departamento de

Agricultura de Estados Unidos 2019).

- Área natural-Áreas (públicas o privadas) con objetivos designados de gestión que incluyen la conservación de la biodiversidad nativa.

Tabla 1. Información adicional de las cinco listas de plantas no autóctonas utilizadas en Florida con situación normativa, usuarios recomendados y enlaces webs para acceder las listas.

\begin{tabular}{|c|c|c|c|}
\hline Lista & $\begin{array}{l}\text { Reguladora } \\
\text { (S/N) }\end{array}$ & Usuarios & Enlace web \\
\hline $\begin{array}{l}\text { Lista Federal de } \\
\text { Malas Hierbas } \\
\text { Nocivas }\end{array}$ & $S$ & $\begin{array}{l}\text { UF/IFAS Profesores y personal, } \\
\text { Programas de Extensión (UF/ } \\
\text { IFAS Programa de Voluntarios } \\
\text { Master Gardeners, Programa de } \\
\text { Paisajismo }{ }^{T M} \text { autóctono de Florida), } \\
\text { administradores de fincas, agencias } \\
\text { federales, estatales y locales, } \\
\text { público en general }\end{array}$ & $\begin{array}{l}\text { https://www.aphis.usda.gov/plant_health/plant_pest_info/weeds/ } \\
\text { downloads/weedlist.pdf }\end{array}$ \\
\hline $\begin{array}{l}\text { Lista de Florida } \\
\text { de Malas } \\
\text { Hierbas Nocivas }\end{array}$ & $S$ & $\begin{array}{l}\text { UF/IFAS Profesores y personal, } \\
\text { Programas de Extensión (UF/ } \\
\text { IFAS Programa de Voluntarios } \\
\text { Master Gardeners, Programa de } \\
\text { Paisajismo }{ }^{T M} \text { autóctono de Florida), } \\
\text { administradores de fincas, agencias } \\
\text { federales, estatales y locales, } \\
\text { público en general }\end{array}$ & https://www.flrules.org/gateway/ruleNo.asp?id=5B-57.007 \\
\hline $\begin{array}{l}\text { La Lista } \\
\text { de Plantas } \\
\text { Acuaticas } \\
\text { Prohibidas en } \\
\text { Florida }\end{array}$ & $\mathrm{S}$ & $\begin{array}{l}\text { UF/IFAS Profesores y personal, } \\
\text { Programas de Extensión (UF/ } \\
\text { IFAS Programa de Voluntarios } \\
\text { Master Gardeners, Programa de } \\
\text { Paisajismo }{ }^{\text {TM }} \text { autóctono de Florida), } \\
\text { administradores de fincas, agencias } \\
\text { federales, estatales y locales, } \\
\text { público en general }\end{array}$ & https://www.flrules.org/gateway/RuleNo.asp?id=5B-64.011 \\
\hline $\begin{array}{l}\text { Lista del Consejo } \\
\text { de Plantas } \\
\text { Exóticas de } \\
\text { Florida (FLEPPC) }\end{array}$ & $\mathrm{N}$ & $\begin{array}{l}\text { Administradores de fincas, agencias } \\
\text { federales, estatales y locales, } \\
\text { público en general }\end{array}$ & https://www.fleppc.org/list/list.htm \\
\hline $\begin{array}{l}\text { Evaluación UF/ } \\
\text { IFAS }\end{array}$ & $\mathrm{N}^{*}$ & $\begin{array}{l}\text { UF/IFAS Profesores y personal, } \\
\text { Programas de Extensión (UF/ } \\
\text { IFAS Programa de Voluntarios } \\
\text { Master Gardeners, Programa de } \\
\text { Paisajismo }{ }^{T M} \text { autóctono de Florida), } \\
\text { administradores de fincas, agencias } \\
\text { federales, estatales y locales, } \\
\text { público en general }\end{array}$ & https://assessment.ifas.ufl.edu \\
\hline \multicolumn{4}{|c|}{$\begin{array}{l}\text { *La Evaluación de UF/IFAS no está regulada para el público en general, pero todo el personal de UF/IFAS que incluye profesores, personal y } \\
\text { programas afiliados (Programa de Voluntarios de maestros de jardinería, Programa de Paisajismo }{ }^{\mathrm{TM}} \text { autóctono de Florida) deben adherirse y } \\
\text { referirse a las conclusiones de esta lista. No se puede recomendar el uso de especies de alto riesgo o invasivas por profesores y personal de UF } \\
\text { IFAS. En ocasiones especiales se aprueba "el uso especifico y limitado" de ciertas especies invasivas de alto riesgo por el grupo de trabajo de } \\
\text { plantas invasivas de UF/IFAS. }\end{array}$} \\
\hline
\end{tabular}

